\title{
RE/POSITIONING AFRICAN MEDIA STUDIES Die Herausforderung, Postkolonialität und Medialität zusammenzudenken
}

von HENRIETTE GUNKEL

Fournal of African Media Studies, hg. v. Winston Mano in Zusammenarbeit mit Monica Chibita und Wendy Willems, erscheint seit Oktober 2009 dreimal jährlich im Intellect Verlag, Bristol.

Fournal of African Cinemas, hg. v. Keyan G. Tomaselli, Martin Mhando, erscheint seit Oktober 2009 halbjährlich im Intellect Verlag, Bristol.

Doris Hegner, Bernd M. Scherer (Hg.), Neues Afrikanisches Kino. Ästhetik und Politik. Betrachtungen von Manthia Diawara (African Film. New Forms of Aesthetics and Politics, 2010), München, Berlin, London, New York (Prestel) 20ı0, übers. von Herwis Engelmann, Petra Huber und Gerd Burger.

Gefordert ist eine kontinuierliche Arbeit am Bild und an Konnotationen, die der negativen Determinierung Afrikas in den westlichen Medien widerstehen - also eine zeitgleich gegenwartsbezogene und zeitlose Vorstellungswelt in Bildern. Das Bild hat mit Geschichte und Gegenwart, aber auch mit Zukunftsweisendem und Revolutionärem zu tun. Kurz und gut: Wir brauchen Bilder, die offen für Interpretationen bleiben und an denen jede Kolonisierung oder absolutistische Definition abprallt. ${ }^{1}$

Der Dokumentarfilm Afro@digital von Balufu Bakupa-Kanyinda ${ }^{2}$ hat bereits 2003 mit dem weit verbreiteten Ansatz aufgeräumt, Medien und digitale Technologien immer erst einmal als westlich zu konzeptualisieren. Das macht er zum einen, indem er das größte Coltan-Vorkommen, materielle Grundlage für fast alle Mikroprozessoren, oder aber das älteste «calculating tool in the world» und damit das mathematische Grund-/Denkmodell für digitale Technologien auf dem afrikanischen Kontinent verortet. Zum anderen eröffnet er die Möglichkeit, neue Fragen zur analogen Datenübertragung zu stellen: danach, wie Technologien angewendet werden und welche Effekte sie dabei erzielen, ${ }^{3}$ auch und vor allem im Hinblick auf Repräsentationsordnungen, Postkolonialitäten, Ästhetiken des Politischen - kurz der Politik der Bilder. Neben der kontinuierlichen Arbeit am Bild und an Darstellungsweisen gerät die Medientechnik selbst mehr und mehr in den Blick. Dabei geht es auch darum, Technologien neu zu formatieren und sie somit dazu zu bringen, andere Dinge zu tun, wie John Akomfrah im Dokumentarfilm argumentiert:

The digital is a technological innovation. But all technology, as we know from the Greek [entelechy], isn't just about tools. The technology is a whole mindset, it's about the ideas, the sensibilities, the way of positioning yourself in relation to the world. All of that, that is technology. [...] So the digital is not simply the machines. It's about a kind of re-formulating the relationship between us with a capital 〈M〉, Man and Machine. And in this repositioning we have the chance to get closer to something that we didn't [get close to] before.

Daraus ergibt sich die Frage, wie technische Möglichkeiten genutzt werden, um andere Bilder, andere Themen, neues Wissen medial zu erfassen und im Endeffekt die Medienund Bildwissenschaften zu dekolonisieren. ${ }^{4}$ Neben Repräsentationsmechanismen und Politiken der Ästhetik geht es dabei auch um den Ansatz, Technologien und Medien selbst postkolonial zu wenden. Dabei ist Postkolonialität nicht nur als Gegenmodell zu begreifen, sondern auch als 
Möglichkeit, wenn nicht gar als Versprechen, losgelöst vom Westen andere Bilder, Produktionen, Diskurse, Technologien und Theorien zu imaginieren. Welche medialen Werkzeuge werden auf dem afrikanischen Kontinent prominent genutzt, um dieses Ziel zu erreichen? Und welche Effekte erzielen sie - auf Darstellungsweisen, Inhalte, Politiken? Welche Rolle spielen bzw. welche Politiken ermöglichen sogenannte neue Medien - ohne gleichzeitig neokoloniale Strukturen zu begünstigen?

\section{I.}

Wie wichtig gerade die sogenannten neuen Medien für ein politisches (Selbst-)Verständnis sowie politische Mobilisierungen sind, zeigen die Ereignisse in Tunesien und Ägypten Anfang 2011. Satellitenfernsehen, Internet, Mobiltelefone, Blogs, Twitter-Feeds, Facebook-Seiten, vielleicht auch WikiLeaks haben die politischen Analysen mit beeinflusst, die Protestbewegungen zum Teil live übertragen, organisiert und multipliziert, auch durch die Dokumentation der brutalen Reaktionen von Seiten der Regierungen. Ägypten, geschult an den Ereignissen in Tunesien, hat nach nur ein paar Tagen der Proteste das Internet blockiert. Die von Luisike Lynete Mukhongos im Titel ihres Aufsatzes für das Journal of African Media Studies formulierte Frage: «Can the media in Africa shape Africa's political future? $\nu^{5}$ ist also bereits zum Teil beantwortet worden, zumindest derzeit für den Kontext der beiden nordafrikanischen Staaten. Gerade in den Ländern, in denen Medien- und Informationspolitiken historisch von den politischen Eliten kontrolliert und monopolisiert worden sind ${ }^{6}$ und somit wenig Raum für eine kritische Masse zugelassen haben, erzielen solch mediale Entwicklungen entscheidende Effekte im Hinblick auf die journalistischen Arbeitsbedingungen, auf Produktion und Weiterleitung von Informationen, Wissen und Bildern, auf die Politik und alternative Medienstrukturen.

Das Ende 2009 erschienene Journal of African Media Studies (JAMS), das sich nicht nur an diesen Ereignissen, sondern auch am Film Afro@Digital messen lassen muss, hat diese Thematiken in verschiedenen Artikeln aufgegriffen; es geht um Anwendungsmöglichkeiten von Medien und neuen Informationstechnologien, um ihre sozialen und politischen Effekte und um die Möglichkeiten, die die Veränderungen für die Platzierung und Theoretisierung von Medienwissenschaften auf dem afrikanischen Kontinent eröffnen. Audrey Gadzekpo z. B. argumentiert, dass die «Re-Demokratisierung〉 vieler postkolonialer Staaten auf dem afrikanischen Kontinent nicht nur zu einer Deregu- lierung und Liberalisierung der Kommunikationssysteme geführt hat, sondern auch zu einem Wiedererstarken von Frauenorganisationen und der damit zusammenhängenden Etablierung von Gender Studies an einigen afrikanischen Universitäten. Und so fordert Gadzekpo eine feministische Medienwissenschaft in einem afrikanischen Kontext, die eine Forschung vorantreibt, welche «Africaspecific data about emerging issues and trends in the media» generieren wird und "at the same time develop new feminist theories that take account of the complex and fluid African condition». ${ }^{7}$ Hier geht es also auch um eine zukünftige Ausrichtung der Medienwissenschaften (und der Geschlechterforschung) im afrikanischen Kontext.

Diese erste Ausgabe von JAMS zielt somit in erster Linie auf ein mapping der Medienwissenschaften auf dem afrikanischen Kontinent, auf die Zusammenarbeit von Wissenschaft und Aktivismus und NGOs, auf Unterschiede und Gemeinsamkeiten zwischen afrikanischen Staaten, auf materielle Grundlagen der tatsächlichen Anwendung von Medien und daran anknüpfend auf die Frage, ob es eine spezifisch afrikanische Medienwissenschaft gibt bzw. geben sollte (wie der Titel des Journals impliziert) und/oder ob das Journal nicht eher eine Medienwissenschaft in Afrika meint, also eine lokale Ausrichtung einer globalen Wissenschaft.

Die Herausgeber von JAMS haben sich auf eine Konzeptionalisierung und Positionierung einer spezifisch afrikanischen Medienwissenschaft verständigt und zielen auf eine Infragestellung der angloamerikanischen Vormachtstellung innerhalb der globalen Media, Communication and Cultural Studies; ${ }^{8}$ 〈Afrika〉 soll aus der medienwissenschaftlichen Peripherie geholt werden. Interessant bei dieser Position ist, dass sie - wie bei vielen Themenfeldern, die

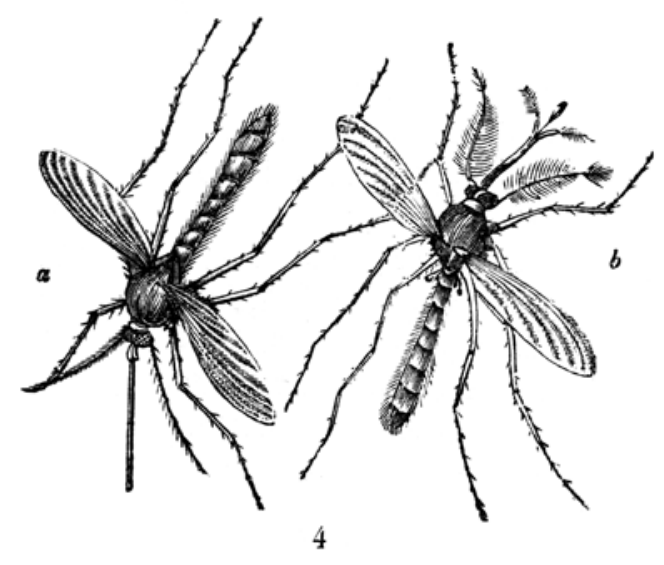



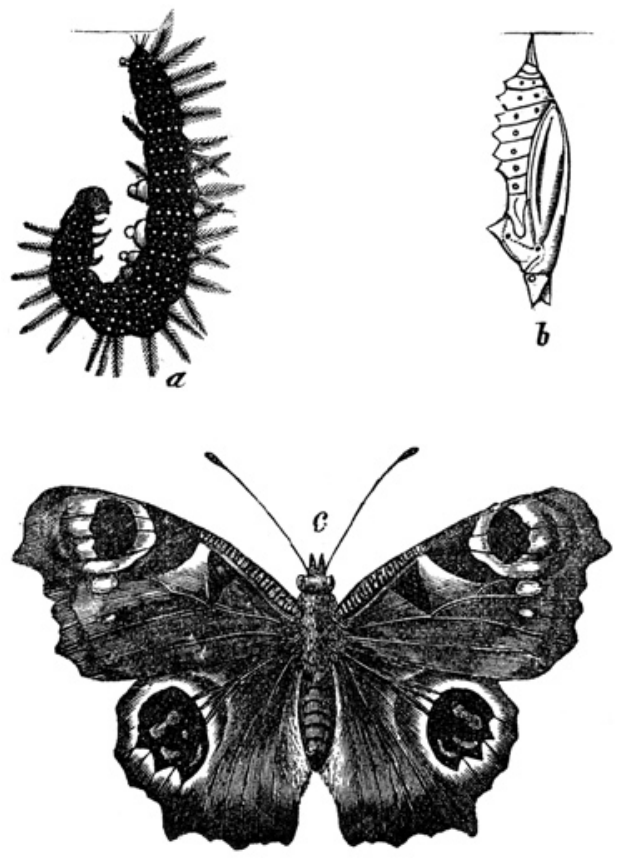

im Kontext Afrikas verhandelt werden - vor allem in Bezug auf einen Mangel und damit in Bezug auf technologische, politische und soziale Veränderungen im Sinne von «Entwicklung verhandelt wird, «to promote the often neglected but important area of media research in Africa», ${ }^{9}$ wie es im Editorial der ersten Ausgabe heißt. ${ }^{10}$ Das heißt, die von Diawara und Akomfrah geforderte Arbeit am Bild und Medium sowie an deren Konnotationen ist hier (noch) nicht berücksichtigt bzw. weniger prominent.

Aber es geht außer um die gängigen Massenmedien auch darum, typisch afrikanische Kommunikationsmedien wie etwa «orale Medien»"11 und/oder «informelle», «kleine» und «indigene» Medien wie «Musik», «Witze» und «Theaten ${ }^{12}$ mit einzubeziehen, wie im Editorial der ersten Ausgabe zu lesen ist. Während diese Medien bisher noch keinen prominenten Platz in den drei Journalausgaben bekommen haben, ist es darüber hinaus fragwürdig, ob diese Erweiterung wirklich sinnvoll ist. Es stellt sich auch die Frage, ob die genannten «Medien» wirklich «indigen» sind oder ob sie nicht eigentlich mediale Prototypen vieler, nicht nur afrikanischer Gesellschaften sind; mit dem Unterschied vielleicht, dass sie in manchen Gesellschaften über einen längeren Zeitpunkt ernst genommen werden als in anderen.
Die Themen in den ersten drei bisher erschienen Ausgaben von JAMS, das vor allem mit dem Africa Media Centre an der University of Westminster assoziiert ist, sind vielfältig: Etwa zum Gebrauch von Mobiltelefonen innerhalb unterschiedlicher Gruppen der nomadischen Communities im Westen Kameruns und die daraus resultierende Neuinterpretation von Raum und Zeit, die sich auch auf soziale Strukturen auswirkt; ${ }^{13}$ die Diskussion um Rugby im heutigen Südafrika, vor allem in Bezug auf race und gender, anhand von Clint Eastwoods Film Invictus (2009); ${ }^{14}$ Kristin Skare Orgerets Analyse der medialen Berichterstattung über Straßennamensänderungen in Durban, die die Herausforderung deutlich macht, die Komplexität und Dynamiken des Post-Apartheid-Staates darzustellen; ${ }^{\mathbf{1 5}}$ Ikechukwu Obiayas empirische Untersuchung über den Konsum von Nollywood-Filmen ${ }^{16}$ im Internet; ${ }^{17}$ Muhammad Jameel Yusha'us empirische Untersuchung des Umgangs der nigerianischen Presse mit dem Themenfeld der Korruption ${ }^{18}$ und Luisike Lynete Mukhongos erwähnte Studie zum Verhältnis von Medien, Zensur und "good governance». ${ }^{19}$ Keyan Tomaselli verweist auf eine Asymmetrie innerhalb der medientechnischen Strukturen - nicht nur in Bezug auf den Westen und Afrika, sondern auch zwischen urbanen und ländlichen Strukturen innerhalb Afrikas selbst. ${ }^{20}$ Dass es bei der Anwendung von Medien immer auch um Produktion von Wissen geht, in diesem Fall ein Wissen über den afrikanischen Kontinent, ist einer der zentralen Punkte, und so geht es in dem Journal eben auch darum, historische und gegenwärtige Gegenbilder zu Diskursen über 〈Afrika〉 zu schaffen und zu diskutieren.

Tomaselli, der das Journal for Africa Cinemas (JAC) herausgibt, das zur gleichen Zeit beim Intellect-Verlag in Bristol erschien wie JAMS und eng mit diesem verwoben ist, ${ }^{21}$ kommt zum folgenden Schluss: «African media studies need to present a picture of itself as always in the making, always engaging, always casting light on other kind of media studies». ${ }^{22}$ Ein anzustrebendes Ziel einer jeden Medienwissenschaft, nicht nur einer afrikanischen. Die Reduzierung dieses Vorhabens auf eine safrikanische Medienwissenschaft) scheint eine Notwendigkeit von «Entwicklung〉 widerzuspiegeln, die auf der Annahme eines Mangels beruht («die Medienwissenschaft auf dem afrikanischen Kontinent ist noch nicht so weit und muss sich - theoretisch, praktisch - noch entwickelnı). Das Bemühen um soziale und politische Veränderungen ist nicht zu trennen von einer Diskurs- und Wissensproduktion, die Postkolonialität auch in Kunst, Architektur, Performance 
oder Experimentalfilm medial zu erfassen sucht und neue Möglichkeiten in Theorie und Praxis erörtert. Dabei genügt meiner Meinung nach ein intensiverer Blick auf die Kunstszenen des Kontinents, vor allem die Videokunst, um eine viel weitere, zukunftsorientierte Politik des Bildes sowie des speziellen Umgangs mit Technologien, wie es Akomfrah beschreibt oder gar fordert, zu erhaschen - ein Blick, der sich allerdings in den bisher erschienenen JAMSAusgaben nicht findet.

\section{II.}

Und so bleiben die in JAMS veröffentlichten Artikel noch hinter den medienwissenschaftlichen Debatten und Studien zurück, die seit Mitte der 199oer Jahre vermehrt zum afrikanischen (Kino-)Film «as the dominant paradigm for recording our realities, [...] as the kind of repository of popular memories», ${ }^{23}$ entstanden sind, in erster Linie mit Blick auf das frankophone Kino. ${ }^{24}$ Und das, obgleich JAMS mit der zweiten Ausgabe ein ganzes Themenheft zum afrikanischen Film bzw. zu Screen Media, wie Guest Editor Lindiwe Dovey es nennt, herausgegeben hat. Auch hier geht es um eine Bandbreite von soziopolitischen Themen und Repräsentationsmodi sowie um Genres (Musical, Autorenkino, Animation) und alternatives Kino (wie z. B. in Ruanda). Damit positionieren sich die beiden Journals (JAMS und JAC) zueinander; die Filmwissenschaft wird als Teil der Medienwissenschaft ernst genommen, die thematischen Überschneidungen sind deutlich.

Das Kino bzw. der Film, der mit dem Kolonialismus auf den afrikanischen Kontinent kam und eng verknüpft mit den kolonialen Projekten und den ihnen zugrunde liegenden Repräsentationsmechanismen und Darstellungsordnungen war, ist im Zuge der Dekolonialisierung kontinuierlich bearbeitet und gewendet worden. Diese Arbeit am Bild ist, wie Akomfrah betont, von Anfang an eng mit der kolonialen Geschichte des Kontinents verknüpft gewesen, die sich meist entweder auf die Aneignung der Vergangenheit durch Erinnerung oder auf das Antizipieren der Zukunft bezieht. Gleichzeitig waren der «process of discovering new identities, new subjectivities, and a new role of the African subject» zentral für das Projekt, das noch heute im weitesten Sinne 〈Afrikanisches Kino〉 heißt. ${ }^{25}$ Dabei geht es bei dem Projekt des «Afrikanischen Kinos〉 nicht um eine beschreibende Benennung, sondern eher um eine (politische und emotionale) Wertung, die sich auf das Filmschaffen von Afrikaner_innen in Afrika und der Diaspora während und nach der Unabhängigkeit bezieht; von heute aus betrachtet bli- cken wir auf mehr als 50 Jahre «Afrikanisches Kino» zurück. Dieses wachsende cultural archive ist im Sinne von Achille Mbembe als Teil der jüngsten Erinnerungen afrikanischer Gesellschaften zu verstehen; «in reading them we can grasp the power of falsification that exists within memory insofar as it bears witness to the experience that the contemporary African subject has of power, language, and life». ${ }^{26}$

Diesem kulturellen Archiv widmet sich Manthia Diawara in einem 2010 erschienenen Buch, herausgegeben von Doris Hegner und Bernd M. Scherer, das einen guten Überblick über das Afrikanische Kino liefert, welches in Deutschland noch recht wenig rezipiert wird. Es verweist darüber hinaus auch auf ein Umdenken und auf neue Trends in Bezug auf das kulturelle Archiv, weg vom Nationalismus und sozialen Realismus, indem es Textbeiträge von wichtigen Filmemachern, Produzenten und Filmwissenschaftlern des Afrikanischen Kinos inkludiert. ${ }^{27}$ Ausgehend vom Filmfestival African Screens im Berliner Haus der Kulturen der Welt, das Diawara 2008 konzeptionierte, nähert er sich nun einer Archäologie der Zukunft des Afrikanischen Films. Angefangen mit einem Rückblick auf den Afrikanischen Film mit dem 40-jährigen Jubiläum des Festival Panafricain du Cinéma et de la Télévision de Ouagadougou (FESPACO), ${ }^{28}$ richtet sich Diawaras Fokus im ersten und gleichzeitig längsten Teil vor allem auf den frankophonen Film, auf das Autorenkino, das Ousmane Sembène entscheidend mitgeprägt hat. Und so ist der unterhaltsame, anekdotenreiche Einstiegs- und Überblickstext (vor allem für ein deutsches Publikum) zum Afrikanischen Kino eine Hommage und eine Abrechnung mit Sembène und seiner «sozialrealistischen Schule» ${ }^{29}$ zugleich.

In einem zweiten Teil versucht Diawara, sich von Sembènes Vermächtnis zu lösen, neue Wege (er nennt es «Wellen») des Afrikanischen Kinos aufzuzeigen und dabei mithilfe von Filmemachern der «neuen Wellen» ein inhaltliches, stilistisches und produktionsbedingtes Selbstverständnis diesbezüglich zu hinterfragen. Das tut er zum einen durch Beiträge von Podiumsdiskussionen während des Filmfestivals und gefilmtem Interviewmaterial, das in Form einer DVD beiliegt. Durch die Historisierung dreier Trends - die Arte-Welle, ${ }^{30}$ die Arbeit der La Guilde des Cinéastes $^{31}$ und das neue afrikanische Erzählkino - unternimmt das Buch einen ernsthaften Versuch des Umdenkens in Bezug auf den Afrikanischen Film, auch indem es den Fokus von der Frage «Was ist Afrika?» zur Frage «Was ist Film?» wendet. ${ }^{32}$ Damit geht es Diawara eben auch darum, 
andere - bei afrikanischen Filmemachern oft unbeliebte - Filmtechniken wie digitale Technologien, Videos oder DVDs mit dem Autorenkino zusammenzubringen. Diawara betont somit nicht nur die vielfältigen Entwicklungen im Afrikanischen Film, sondern bezieht auch die Videoproduktionen Nigerias, Ghanas und Kenias ein - vor allem die Filmindustrie Nollywoods, der Diawara den dritten Abschnitt im Buch widmet. Damit historisiert und verkompliziert Diawara das Konzept des Afrikanischen Kinos und den dem Konzept zugrunde liegenden Erfahrungsraum (der über eine längere Zeit auch mit Begriffen wie «Drittes Kino〉, 〈Schwarzes Kino» und 'Welt-Kino verbunden war).

Während Diawara das in letzter Konsequenz meiner Meinung nach nur zum Teil gelingt, was allein schon in der unterschiedlichen Gewichtung seiner Sektionen sichtbar wird, sind seine Interviewpartner ${ }^{33}$ schon deutlicher, allen voran John Akomfrah, der die Debatte um das Afrikanische Kino vor allem im Hinblick auf die post-kinematische Realitäten vieler afrikanischer Länder diskutiert:

The fact is that through these avenues - video, digital, DVD - the majority of people on this continent are getting access to their image. [...] If you subject Nollywood [...] to the scrutiny ... of cinema there is failure. But if you turn the table around and you say swhat is really the cinema for? ? and if the answer to that is it is to provide images of a culture to itself, then these socalled inferior formats are succeeding, particularly at the point where the cinema failed. And it is not good enough anymore to endlessly talk about African cinema anymore. It is not good enough to talk about the image in Africa always in the language of emergency or crisis. Because to be honest with you there are places where there is no crisis. [...] If you go to urban Lagos or urban Accra, these people don't feel they're missing images of themselves.

Akomfrah historisiert somit den Begriff und das Konzept des Afrikanischen Kinos, das er direkt mit dem auch emotionalen Projekt der filmischen Suche nach postkoIonialen Gegenbildern vom afrikanischen Kontinent und seiner Geschichte(n) sowie nach neuen Subjektpositionen verbindet. Dabei betont Akomfrah die Bedeutungsverlagerung in der Diskussion um das Bild, die durch die Ablösung vom Kino als ehemals wichtigstem Medium im Zusammenhang mit Repräsentation durch andere Formen des Films, wie Video und DVD, entsteht. Während das Afri- kanische Kino vorher die Diskussion um die Möglichkeiten des Bildes in dem Sinne beeinflusste, dass das Kino Wege eröffnete, andere Subjektpositionen und Utopien zu imaginieren und zu schaffen (die oft wie im Falle Sembènes sozialrealistisch waren), so gibt es heute durch Nollywood und ähnliche Videoproduktionen eine Gleichzeitigkeit von verschiedenen Utopien durch Film, die wir laut Akomfrah heute anders konzipieren und benennen müssen, auch und gerade weil das Image vom afrikanischen Subjekt und seine Geschichte inzwischen medial zugänglich sind, zumindest im urbanen Afrika.

Dass sich Filmwissenschaftler_innen schwer damit tun, diese Bedeutungsverlagerung im Afrikanischen Film zuzulassen und zu theoretisieren, zeigt sich auch an den beiden Journals JAMS und JAC, wo sie in der Konzeption und Ausrichtung (noch) keine Berücksichtigung findet. Die Pluralsetzung z. B. im Titel des Journal of African Cinemas ist ein gängiges Mittel, um auf die (geografischen, stilistischen, technischen, politischen) Diversitäten hinzuweisen, vermittelt aber gleichzeitig eine Art Hilflosigkeit. Ein Effekt dieser Hilflosigkeit ist, dass bei allem Bemühen, die Arbeit von afrikanischen Filmemacher_innen prominenter zu diskutieren, diese weiterhin vor allem im Kontext Afrikas diskutiert werden und nicht als Teil einer internationalen Filmproduktion. Nichtsdestotrotz hat JAC, anders als das Buchprojekt Diawaras, die Möglichkeit, die ganze Breite des Filmschaffens auf dem afrikanischen Kontinent zu dokumentieren und zu diskutieren, nicht nur aus einem sozio-politischen oder produktions- und distributionsbezogenen Interesse heraus, sondern auch im Hinblick auf die Medialität selbst, und zwar innerhalb einer internationalen, nicht nur afrikanischen, Film- und Medientheorie. Damit wäre nicht nur eine Arbeit an den «Konnotationen, die der negativen Determinierung Afrikas in den westlichen Medien widerstehen", ${ }^{34}$ dokumentiert, sondern auch eine kontinuierliche Arbeit am Bild.

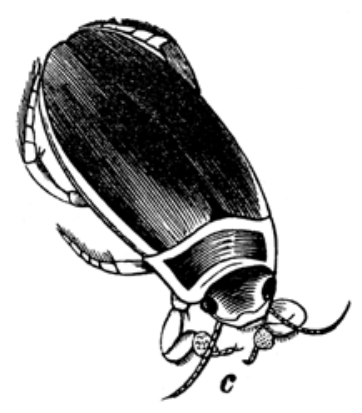


1 Manthia Diawara in: Doris Hegner, Bernd M. Scherer (Hg.), Neues Afrikanisches Kino. Ästhetik und Politik. Betrachtungen von Manthia Diawara, 13 of.

2 Afro@digital, Regie: Balufu Bakupa-Kanyinda, Kongo/Frankreich 2003.

3 John Akomfrah z. B. spricht vom Ende der klassischen Sensitometrie und der ihr zugrunde liegenden Privilegierung weißer Hautfarbe als Ausdruck «klassischen Schönheit im Film durch die Möglichkeiten des Farbfilms seit den $1950 e r J a h r e n$.

4 Ulrike Bergermann unterscheidet zwei Ebenen postkolonialer Medientheorie: zum einen die Analyse von medialen Funktionen und Inhalten in kolonialen und postkolonialen Kontexten; zum anderen die Untersuchung der Möglichkeitsbedingungen von Darstellung, die sich nur in Bezug auf ein Anderes herausgebildet hat; Ulrike Bergermann, PostkoIoniale Medienwissenschaft, in: Julia Reuter, Alexandra Karentzos (Hg.), Schlüsselwerke der Postcolonial Studies, Wiesbaden (VS Verlag) 2011, i. Dr.

5 Luisike Lynete Mukhongo, Can the media in Africa shape Africa's political future?, in: Journal of African Media Studies, Vol. 2, Nr. 3 , 2010, 339.

6 Nur ein Beispiel: Das Apartheitsregime in Südafrika hat erst 1976 das Fernsehen zugelassen.

7 Audrey Gadzekpo, Missing links: African media studies and feminist concerns, in: Journal of African Media Studies, Vol. 1, Nr.1, $2009,78$.

8 Ein Vorhaben, in dem Sprache keine unbedeutende Rolle spielt - und doch werden Artikel beider Journals ausschließlich auf Englisch publiziert (immerhin nur eine von drei Kolonialsprachen), während kaum ein Text aus einer anderen Sprache übersetzt wurde.

9 Editorial, Positioning African Media Studies, in: Journal of African Media Studies, Vol. 1, Nr. 1, 2009, 3.

10 Ähnliche Projekte und

Debatten innerhalb der Cultural/ Media Studies findet man auch in anderen geografischen Kontexten, zum Beispiel im asiatischen Raum; siehe zum Beispiel die Zeitschrift
Journal of Inter-Asia Cultural Studies (Taylor \& Francis), hg. u. a. von Chua Beng Huat und Chen Kuanhsing (diesen Hinweis verdanke ich Markus Stauff).

11 Editorial, Positioning African Media Studies, 4.

12 Ebd., 3.

13 Mirjam de Bruijn, Francis Nyamnjoh, Tseghama Angwafo, Mobile interconnections: Reinterpreting distance, relating and difference in the Cameroonian Grassfields, in: Journal of African Media Studies, Vol. 2, Nr. 3, 2010, 267-285.

14 Louise Vincent, Sasha Stevenson, Rethinking rugby and the rainbow nation, in: Journal of African Media Studies, Vol. 2, Nr. 3 , 2010, 287-296.

15 Kristin Skare Orgeret, The road to renaming - what's in a name? The changing of Durban's street names and its coverage in The Mercury, in: Journal of African Media Studies, Vol. 2, Nr. 3, 2010, 297-320.

16 Unter Nollywood versteht man die nigerianische Film- und Videoproduktionen, die vor allem seit den 199oern enorm gewachsen sind und Nigeria mittlenweile zur zweitgrößten Filmnation der Welt gemacht haben (nach Indien/ Bollywood).

17 Ikechukwu Obiaya, Nollywood on the internet: A preliminary analysis of an online Nigerian video-film audience, in: Journal of African Media Studies, Vol. 2, Nr. 3 , 2010, 321-338.

18 Muhammad Jameel Yusha'u, Regional parallelism and the reporting of corruption in the Nigerian press, in: Journal of African Media Studies, Vol. 2, Nr. 3, 2010, 353-369.

19 Mukhongo, Can the media in Africa shape Africa's political future?, 339-352.

20 Keyan G. Tomaselli, Repositioning African media studies: thoughts and provocations, in: Journal of African Media Studies, Vol.1, Nr. 1, 2009, 9-21.

21 So ist Tomaselli zum Beispiel auch Mitglied des Editorial Boards von JAC. Außerdem bringt er das Journal Critical Arts: A Journal of South-North Cultural Studies heraus und positioniert sich dadurch momentan international im Feld der Medien- und Bildwissenschaft Afrikas.

22 Tomaselli, Repositioning African Media Studies, 18.

23 John Akomfrah in: Afro@ digital, Kongo/Frankreich 2003.

24 Der Fokus auf das frankophone (Autoren-)Kino ist vor allem der Tatsache geschuldet, dass das Kino der Länder der sogenannten Frankophonie West- und Zentralafrikas auch nach der Unabhängigkeit von der französischen Industrie dominiert wurde und international einfacher zugänglich war. Als französische Co-Produktionen waren sie eher auf internationalen Festivals zu sehen als Filme aus Ghana und Nigeria, die ohne quasistaatliche Unterstützung auskommen mussten. Auch die Verbreitung über den französischdeutschen TV-Kanal Arte wurde durch die Tatsache erleichtert, dass die Redaktionen des Senders als Koproduzenten französische Regierungsgelder in afrikanische Produktionen stecken konnten. Das so produzierte Kino war und ist eher auf internationalen Filmfestivals und in internationalen Kulturkanälen zu sehen als in den lokalen afrikanischen Kinos und den TV-Stationen vor Ort. Das Kino im anglophonen, aber vor allem dem lusophonen Afrika ist ein eher wenig bearbeitetes Feld und beschränkt sich meist auf Artikel über die jeweilige nationale Filmproduktion. Ausnahme ist die stetig wachsende Literaturliste zu Nollywood. Ähnlich steht es mit der wissenschaftlichen Bearbeitung der (Apartheids- und Post-Apartheids-) Filmindustrie Südafrikas. Abhandlungen beider Filmindustrien beziehen jedoch selten die politischen und kulturell-historischen Transferprozesse innerhalb des anglophonen Afrikas mit ein.

25 John Akomfrah in: Afro@ digital.

26 Achille Mbembe, Subject and Experience, in: Nadia Tazi (Hg.), Keywords/Experience. For a Different Kind of Globalization, Johannesburg (Double Storey Books) 2004, 18.

27 Das Buch knüpft an seine eigene Arbeit als Filmwissenschaftler und Filmemacher an, siehe z. B. Manthia Diawara, African Cinema:
Politics and Culture, Bloomington (Indiana University Press) 1992.

28 Ousmane Sembène wird allgemein als «Vater des Afrikanischen Kinos' gesehen, vgl. dazu Annett Busch, Max Annas (Hg.), Ousmane Sembène: Interviews, Jackson (University Press of Mississippi) 2008.

29 Diawara in Hegner, Scherer (Hg.), Neues Afrikanisches Kino, 145.

30 Die «Arte-Welle` ermöglichte es Filmemachern, Filme (mit kleinem Budget, kleinen Kameras) für Arte zu drehen, «ohne sich um Zuschauerquoten und künstlerische, politische oder philosophische Vorgaben kümmern zu müssen.» Dazu zählen Filmemacher wie Gahite Fofana, Mahamat-Saleh Haroun, Issa Serge Coelo und Abderrahmane Sissako; Diawara in Hegner, Scherer (Hg.), Neues Afrikanisches Kino, 100.

31 Ein Verbund, der eine neue politische Filmsprache im Grenzbereich zwischen Dokumentarund Experimentalfilm auch in Bezugnahme auf die afrikanische Diaspora fordert, um darauf zu bestehen, dass «die Bildwelten Afrikas und seiner Diaspora unentwirrbar verflochten sind»; Diawara in Hegner, Scherer (Hg.), Neues Afrikanisches Kino, 130 - wie etwa Akomfrahs Handsworth Songs (1986), Jihan el-Tahris Cuba: An African Odysee (2003) oder Balufu Bakupa-Kanyindas Juju Factory (2007). Daneben werden Jean-Marie Téno, Mama Keïta, Dany Kouyaté und Jean-Pierre Bekolo genannt; Diawara in Hegner, Scherer (Hg.), Neues Afrikanisches Kino, 120.

32 Diawara in Hegner, Scherer (Hg.), Neues Afrikanisches Kino, 98.

33 Das Interviewmaterial liegt gekürzt in Form einer DVD bei, auch ein Film wurde aus den Interviews geschnitten. Von einigen der interviewten Filmemacher_innen ist im Anhang des Buches noch die jeweilige Filmografie zu finden.

34 Diawara in Hegner, Scherer (Hg.), Neues Afrikanisches Kino, 130. 